JOURNAL of

MAINE MEDICAL CENTER Journal of Maine Medical Center

Volume 2

Issue 2 Volume 2, Issue 2 (July 2020)

Article 17

2020

\title{
Lessons From an Evidence-Based Medicine Exploration: When Transparency and Sensitivity (Analyses) Can Change the Take- Home Message
}

Nadi N. Kaonga

Maine Medical Center

Et al.

Follow this and additional works at: https://knowledgeconnection.mainehealth.org/jmmc

Part of the Medical Education Commons, and the Obstetrics and Gynecology Commons

\section{Recommended Citation}

Kaonga, Nadi N. and Fitzgerald, Toby (2020) "Lessons From an Evidence-Based Medicine Exploration: When Transparency and Sensitivity (Analyses) Can Change the Take-Home Message," Journal of Maine Medical Center. Vol. 2 : Iss. 2 , Article 17.

Available at: https://knowledgeconnection.mainehealth.org/jmmc/vol2/iss2/17 https://doi.org/10.46804/ 2641-2225.1055

The views and thoughts expressed in this manuscript belong solely to the author[s] and do not reflect the opinions of the Journal of Maine Medical Center or MaineHealth.

This Commentary is brought to you for free and open access by Maine Medical Center Department of Medical Education. It has been accepted for inclusion in the Journal of Maine Medical Center by an authorized editor of the MaineHealth Knowledge Connection. For more information, please contact Dina McKelvy mckeld1@mmc.org.

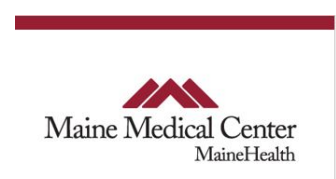




\section{Lessons From an Evidence-Based Medicine Exploration: When Transparency and Sensitivity (Analyses) Can Change the Take-Home Message}

\section{Acknowledgements}

The authors would like to thank the following individuals and departments for their insights and contributions throughout the evidence-based medicine exploration: Dr Wendy Craig, Dr Deborah Sepulveda, Ms Sarah Austin, the MMC Billing Department, the MMC Pharmacy and the Business Intelligence team.

\section{Authors}

Nadi N. Kaonga and Toby Fitzgerald 


\title{
Lessons from an Evidence-Based Medicine Exploration: When Transparency and Sensitivity (Analyses) Can Change the Take-Home Message
}

\author{
Nadi N Kaonga, MD, MHS, MS, ${ }^{1}$ Toby Fitzgerald, DO ${ }^{1}$ \\ ${ }^{1}$ Department of Obstetrics and Gynecology, Maine Medical Center, Portland, ME
}

\section{BACKGROUND}

Induction of labor is quite common in the United States. While induction rates have declined over the last decade, nearly 1 in 4 deliveries $(23 \%)$ in the United States is induced for medical or elective reasons. ${ }^{1}$ Of the induction agents available, intravenous (IV) oxytocin is used in an estimated $25 \%$ of cases. ${ }^{2}$ At our academic medical center, $40 \%$ of our labor inductions from April 2019 to September 2019 used IV oxytocin (unpublished data). This medication comes with high risks, including uterine tachysystole, hyperstimulation, fetal distress, hypotension, hypertension, hyponatremia, seizures, and coma. ${ }^{2}$

To understand our use of IV oxytocin and its impact on birth outcomes at our institution, we conducted a literature search and discussed the findings in our department's evidence-based medicine (EBM) session (ie, journal club). We identified 4 recent articles that performed systematic reviews evaluating birth outcomes and use of IV oxytocin. In particular, the articles examined how discontinuing IV oxytocin during the active phase of labor impacts the cesarean section (C-section) rate. ${ }^{2,3-5}$ While all 4 review articles concluded that discontinuing IV oxytocin in the active phase of labor significantly reduced the $\mathrm{C}$-section rate, 1 article conducted sensitivity analyses that, importantly, changed the interpretation of the primary outcome. ${ }^{2}$ With this new interpretation, we identified important considerations when reviewing the literature. In this article, we share our lessons from our EBM exploration on this topic to help inform more

Correspondence: Nadi N Kaonga MD, MHS, MS

Maine Medical Center

Department of Obstetrics and Gynecology

22 Bramhall St, Portland, Maine 04102

nkaonga@mmc.org rigorous and creative approaches to interpreting the literature.

\section{LESSONS}

Know your resources and think outside of the box

In our Department of Obstetrics and Gynecology, our method for EBM literature reviews is to first identify a faculty mentor and also consider meeting with the department's research navigator. The research navigator has a background in research methods, biostatistics, and epidemiology. Given their skillset, they serve as a resource to better understand the clinical literature. Our department's research navigator helped explain and clarify the more advanced statistical concepts in the review articles. In our process, they also encouraged nontraditional thinking and prompted our exploration of the supply and distribution of IV oxytocin at our institution.

In this pursuit, we spoke with the pharmacy and billing departments to better understand how IV oxytocin is acquired and distributed, and what it costs our institution and patients. To learn more and contextualize our center's use of IV oxytocin, we worked with our department's head of quality improvement to compile data and analyze births and inductions over a 6month period. This approach allowed us to conceptualize the review articles' findings within our local context.

The research navigator, pharmacy staff member and billing representative supported the exercise, and their additional input helped to enrich the subsequent EBM discussion with practical cases and considerations. We were also able to supply evidence (albeit specific to our academic medical 
Journal of Maine Medjcal Cen center) that was otherwise missing from the globa evidence base for discussion.

\section{Read more than one article}

For the purpose of our department's EBM session, we selected 1 review article to focus on and discuss. While we had 1 article in mind, we conducted a cursory review in PubMed that helped identify 4 similar review articles. Without this cursory review, we would not have found the article by Boie et al. ${ }^{2}$ There was $78 \%$ to $100 \%$ overlap of references between the 4 review articles, and we were able to read the additional articles to compare methods and findings. This process revealed a crucial, yet overlooked, limitation and analysis in 3 of the review articles that ultimately changed the interpretation of the primary outcome. Boie and colleagues took these factors into consideration in their review.

\section{Checklists and reporting guidelines are a helpful start}

In 2 of the most recent 4 review articles, ${ }^{2,5}$ the Preferred Reporting Items for Systematic Reviews and Meta-Analyses (PRISMA) Checklist was used to guide the reading and appraisal of the articles. ${ }^{6}$ Both articles accounted for all items within the text or supplementary files. However, only through closer reading and comparison did we understand how and why only Boie et al conducted sensitivity analyses in their review article.

\section{Transparency matters}

Boie and colleagues were very transparent in their reporting. They justified why they conducted specific types of analyses. Oftentimes, their analyses were limited by the level of reporting in the individual studies. Accordingly, they contacted authors of the individual studies to obtain as much detail as possible regarding the included data.

\section{Sensitivity analyses can be game changers}

Sensitivity analyses provide the ability to assess the robustness of a primary result and test how the results may or may not hold based on different assumptions. ${ }^{7}$ The sensitivity analysis conducted by Boie and colleagues revealed a critical omission in previous analyses of the primary outcome. While all the review articles concluded that the C-section rate was significantly reduced when IV oxytocin was discontinued during the active phase of labor, the sensitivity analysis in the Boie et al review only included data on $\mathrm{C}$-sections after the active phase. By focusing only on these patients, they found that discontinuing IV oxytocin in the active phase

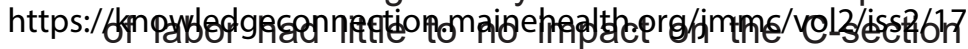
DOI: $10.46804 / 2641-2225.1055$ rate. This fis finding 2 Art. 17 underscores the importance of understanding assumptions and assessing data in different ways. Furthermore, the sensitivity analysis conferred a greater level of certainty (moderate) but only reflected data from 4 studies. When considering practice, this finding was now less influential, and it also resulted in closer critique and questioning of the findings for the secondary outcomes.

Additionally, IV oxytocin is a high-risk medication with several severe side effects. While its continuation minimally reduces the time from labor to delivery and its discontinuation does not impact the C-section rate, a risk-benefit analysis for each use case must be considered.

\section{CONCLUSION}

Ultimately, EBM discussions are meant to challenge and/or inform practice. The lessons learned from this EBM exploration are salient reminders that data reported in articles should not be blindly accepted. We hope that the lessons we shared in this article will serve as a helpful starting point and approach to more rigorous and creative EBM reflection and discussions.

Keywords: obstetrics, oxytocin, induction, labor, evidence-based medicine

\section{Conflicts of Interest: None}

\section{REFERENCES}

1. Osterman MJK, Martin JA. Recent declines in induction of labor by gestational age. National Center for Health Statistics Data Brief No. 155, June 2014. Accessed January 20, 2020. https:// www.cdc.gov/nchs/products/databriefs/db155.htm.

2. Boie S, Glavind J, Velu AV, et al. Discontinuation of intravenous oxytocin in the active phase of induced labor. Cochrane Database Syst Rev. 2018;8(8);CD012274. doi:10.1002/14651858. CD012274.pub2.

3. Vlachos DE, Pergialiotis V, Papantoniou N, Trompoukis $\mathrm{S}$, Vlachos GD. Oxytocin discontinuation after the active phase of labor is established. J Matern Fetal Neonatal Med. 2015;28(12):1421-1427. doi:10.3109/14767058.2014.955000.

4. Saccone G, Ciardulli A, Baxter J, et al. Discontinuing oxytocin infusion in the active phase of labor: a systematic review and meta-analysis. Obstet Gynecol. 2017;130(5):1090-1096. doi:10.1097/AOG.0000000000002325.

5. Hernández-Martínez A, Arias-Arias A, Morandeira-Rivas A, Pascual-Pedreño AI, Ortiz-Molina EJ, Rodriguez-Almagro J. Oxytocin discontinuation after the active phase of induced labor: a systematic review. Women Birth. 2019;32(2):112-118. doi:10.1016/j.wombi.2018.07.003.

6. Moher D, Liberati A, Tetzlaff J, Altman DG, PRISMA Group. Preferred reporting items for systematic reviews and meta-analyses: the PRISMA Statement. PLoS Med. 2009;6(7):e1000097. doi:10.1371/journal.pmed.1000097.

7. Thabane L, Mbuagbaw L, Zhang S, et al. A tutorial on sensitivity analyses in clinical trials: the what, why, when and how. $B M C$ Med Res Methodol. 2013;13:92. doi:10.1186/1471-2288-13-92. 